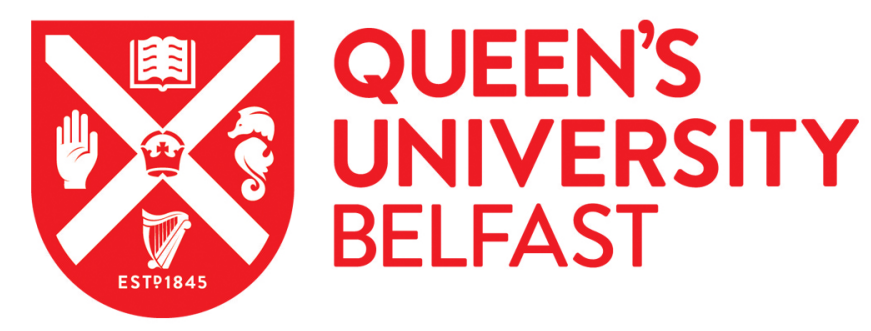

\title{
Nonlinear frequency coupling in dual radio-frequency driven atmospheric pressure plasmas
}

Waskoenig, J., \& Gans, T. (2010). Nonlinear frequency coupling in dual radio-frequency driven atmospheric pressure plasmas. Applied Physics Letters, 96(18), 181501-181501-3. [181501].

https://doi.org/10.1063/1.3425668

Published in:

Applied Physics Letters

Document Version:

Publisher's PDF, also known as Version of record

Queen's University Belfast - Research Portal:

Link to publication record in Queen's University Belfast Research Portal

Publisher rights

(C) 2010 American Institute of Physics

This work is made available online in accordance with the publisher's policies. Please refer to any applicable terms of use of the publisher.

\section{General rights}

Copyright for the publications made accessible via the Queen's University Belfast Research Portal is retained by the author(s) and / or other copyright owners and it is a condition of accessing these publications that users recognise and abide by the legal requirements associated with these rights.

Take down policy

The Research Portal is Queen's institutional repository that provides access to Queen's research output. Every effort has been made to ensure that content in the Research Portal does not infringe any person's rights, or applicable UK laws. If you discover content in the Research Portal that you believe breaches copyright or violates any law, please contact openaccess@qub.ac.uk. 


\title{
Nonlinear frequency coupling in dual radio-frequency driven atmospheric pressure plasmas
}

\author{
J. Waskoenig ${ }^{\text {a) }}$ and T. Gans \\ Centre for Plasma Physics, Queen's University Belfast, Belfast BT7 1NN, Northern Ireland, \\ United Kingdom
}

(Received 27 February 2010; accepted 12 April 2010; published online 3 May 2010)

\begin{abstract}
Plasma ionization, and associated mode transitions, in dual radio-frequency driven atmospheric pressure plasmas are governed through nonlinear frequency coupling in the dynamics of the plasma boundary sheath. Ionization in low-power mode is determined by the nonlinear coupling of electron heating and the momentary local plasma density. Ionization in high-power mode is driven by electron avalanches during phases of transient high electric fields within the boundary sheath. The transition between these distinctly different modes is controlled by the total voltage of both frequency components. (c) 2010 American Institute of Physics. [doi:10.1063/1.3425668]
\end{abstract}

Dual radio-frequency driven plasmas, with two independent power sources, attract rapidly growing attention motivated by increased opportunity for plasma manipulation and additional phenomena due to nonlinear frequency coupling. ${ }^{1-8}$ Fundamental insight is essential for the exponentially rising interest in homogeneous non-thermal plasmas at ambient pressure. ${ }^{9-14}$ These plasmas are highly susceptible to instabilities initiated through mode transitions and associated variations in plasma ionization mechanisms. ${ }^{15,16}$ Frequency coupling in such systems significantly increases complexity; however it also promises additional manipulation to face the major challenge of stabilization and control.

The role of frequency coupling in the dynamics of plasma ionization and associated mode transitions is investigated using numerical simulations of an ambient pressure plasma with dual frequency operation. Two sinusoidal voltage signals are applied across a discharge gap between two equal area electrodes; $V=V_{\mathrm{lf}} \cos \left(2 \pi f_{\mathrm{lf}} t\right)+V_{\mathrm{hf}} \cos \left(2 \pi f_{\mathrm{hf}} t\right)$. Here, $V$ is the applied voltage signal, $V_{\mathrm{lf}}, f_{\mathrm{lf}}, V_{\mathrm{hf}}$, and $f_{\mathrm{hf}}$ are the voltage amplitude and frequency of the low frequency (lf) and the high frequency (hf) component, respectively.

The electrode dimensions are assumed to be large in comparison to the discharge gap, allowing one-dimensional treatment. The employed numerical model is based on hydrodynamic equations with semikinetic treatment of electrons. Rates for electron impact reactions as well as the mobility and diffusion constant of electrons are determined with the Boltzmann solver BOLSIG+ (Ref. 17) and stored in look-up tables versus the mean electron energy. This approach is valid under atmospheric pressure conditions since the mean free path of electrons is small compared to the Debye length. Recent single frequency experiments probing the excitation and ionization dynamics show excellent agreement. ${ }^{18}$

The plasma chemistry is kept as simple as possible to provide the best possible insight into details of the frequency coupling. The simplest system of experimental relevance is a helium atmosphere with a small nitrogen admixture $(0.1 \%)$ as an impurity. Nitrogen impurities have been found to be of major importance, since Penning ionization by metastable excited particles is a crucial ionization mechanism under am-

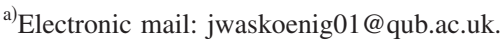

bient pressure conditions. ${ }^{19}$ Eight species, namely, neutral helium $(\mathrm{He})$, excited helium $\left(\mathrm{He}^{*}\right)$, excited helium molecules $\left(\mathrm{He}_{2}^{*}\right)$, helium positive ions $\left(\mathrm{He}^{+}\right)$, helium molecular ions $\left(\mathrm{He}_{2}^{+}\right)$, nitrogen $\left(\mathrm{N}_{2}\right)$, nitrogen ions $\left(\mathrm{N}_{2}^{+}\right)$, and electrons $(e)$ are considered. The secondary electron emission coefficient $\gamma$ is set to 0.25 for helium species and 0.005 for nitrogen ions. ${ }^{15}$ The neutral background gas temperature is assumed to be $300 \mathrm{~K}$.

The governing equations are the continuity equation for electrons, ions, and neutrals using the drift diffusion approximation, Poisson's equation and electron energy conservation. A detailed description of the set of equations and boundary conditions can be found in Ref. 20 and references therein.

Investigations of single frequency plasmas have shown multiple solutions of steady state plasma for the same applied voltage. This mode transition represents the distinctly different constricted $\gamma$-mode and the controlled $\alpha$-mode. ${ }^{15,16}$ Unique solutions can, in principle, be obtained through setting the current rather than the voltage. These two approaches are, however, not equivalent. The nonlinear character of the plasma boundary sheath inherently generates higher harmonics, which are experimentally observed in the current rather than the applied voltage. ${ }^{21}$ This requires setting a sinusoidal voltage signal rather than the current and selfconsistently solving the generated higher harmonics in the current. Unique solutions are obtained by fixing the power coupled into the plasma. ${ }^{15}$

For the following investigations a discharge gap of 2.4 $\mathrm{mm}$ (comparable to Yuan et al. ${ }^{22}$ ) is chosen. The applied frequencies are 2 and $14 \mathrm{MHz}$ (comparable to O'Connell et $\left.a l .{ }^{6}\right)$. Consequences of frequency coupling are revealed in details of the ionization and excitation dynamics of the plasma. Similar to single frequency investigations ${ }^{22}$ it is found that Penning ionization of nitrogen by helium metastables $\left(\mathrm{He}^{*}+\mathrm{N}_{2} \rightarrow \mathrm{He}+\mathrm{N}_{2}{ }^{+}+e\right)$ is the dominant ionization process. It exceeds the direct electron impact ionization of helium $\left(e+\mathrm{He} \rightarrow \mathrm{He}^{+}+2 e\right)$ at low input powers even by an order of magnitude. Penning ionization is inherently correlated with the production of helium metastables $\left(\mathrm{He}^{*}\right)$ mainly driven by electron impact excitation of helium $\left(e+\mathrm{He} \rightarrow \mathrm{He}^{*}+e\right)$.

The time and space resolved electron impact excitation of helium for low power operation at $V_{\text {lf }}=80 \mathrm{~V}$ and 


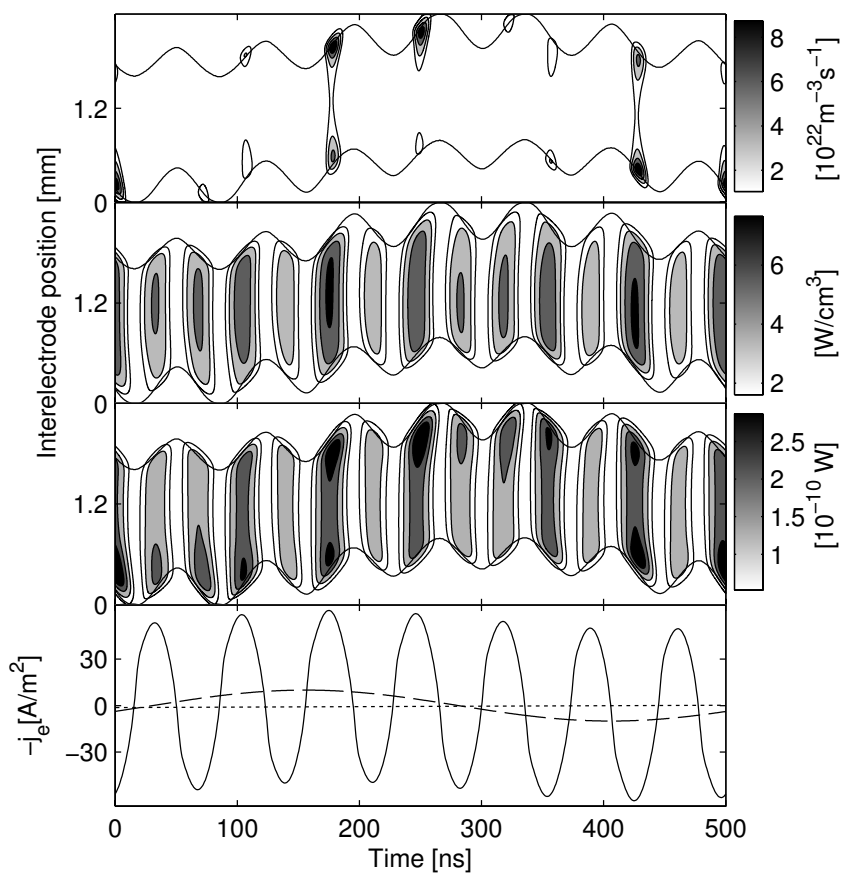

FIG. 1. Time and space resolved electron impact excitation of helium (a), power absorbed by the electrons $\left(j_{e} \cdot E\right)$ (b), absorbed power per charged particle (c), and the negative electron current density in the discharge center (d) at $V_{\mathrm{lf}}=80 \mathrm{~V}$ and $P_{\mathrm{set}}=0.5 \mathrm{~W} / \mathrm{cm}^{2}$.

$P_{\text {set }}=0.5 \mathrm{~W} / \mathrm{cm}^{2}$ is plotted on a linear gray scale in Fig. 1(a). The solid line indicates the boundary between the plasma bulk and the plasma boundary sheaths, which is determined assuming an equivalent sharp electron step. ${ }^{23} \mathrm{Un}$ der these conditions the plasma operates in the controlled $\alpha$-mode, where the excitation mechanisms are volumetric. This mode is mainly driven by the electron current density, leading to pronounced excitation structures during sheath expansion and sheath collapse. The sheath collapse structure is induced by a transient localized reversal of the electric field, which can be explained through the collision dominated environment present under atmospheric pressure conditions. The electrons cannot instantaneously respond to the rapidly changing plasma boundary potential due to collisions with the background gas. This causes a charge accumulation resulting in an electric field accelerating electrons toward the electrodes. Compared to single frequency excitation ${ }^{18}$ one can observe several sheath expansion and sheath collapse structures per $2 \mathrm{MHz}$ cycle, which vary in intensity. The excitation is most efficient at 180 and at $430 \mathrm{~ns}$. Here the electron current density has a global extremum [Fig. 1(d)], where it shows local extrema in the less efficient excitation structures. Due to current continuity electrons are faster in regions of low electron densities closer to the electrodes. This corresponds to more pronounced field reversal structures compared to the sheath expansion at 180 and $430 \mathrm{~ns}$.

Figure 1(b) shows the time and space resolved power absorbance of the electrons $\left(j_{e} \cdot E\right)$ on a linear gray scale. There is a strong correlation to the electron current density [Fig. 1(d)], which also has global extrema at 180 and $430 \mathrm{~ns}$. Spatially all power absorption structures are maximum in the plasma center. However, the electron impact excitation is maximum near the plasma boundary sheaths. This disparity is due to the nonlinearity of the electron impact excitation process. It depends linearly on electron density but over-



FIG. 2. Time and space averaged absorbed power by the electrons $\left(j_{e} \cdot E\right)$ as well as time averaged total electron production rate in the plasma volume $\left(S_{e}\right)$ (a). Electron density in the plasma bulk $\left(n_{e}\right)$ and electron flux at the electrodes $\left(\Gamma_{e}\right)$ (b) under variation in the if voltage amplitude at a fixed power density of $P_{\text {set }}=0.5 \mathrm{~W} / \mathrm{cm}^{2}$.

linearly on the electron energy distribution. Thus, the excitation is more critically determined by the power absorbed per electron rather than the total power. This is illustrated in Fig. $1(\mathrm{c})$. Here $j_{e} \cdot E$ is divided by the ion density, which is quasiequal to the electron density within the plasma bulk. In this way, division by zero within the momentary plasma boundary sheaths is avoided. The power absorbed per electron is maximum near the boundary sheaths comparable to the excitation structures [Fig. 1(a)]. Here the electron density is lower than in the plasma center leading to a higher power absorbance of each electron. The $2 \mathrm{f}$ operation allows tailoring the $j_{e} \cdot E$ structures in certain phases to regions of lower electron densities leading to a higher absorbance per electron. This explains, that the sheath collapse structure at 230 ns shows a higher excitation rate than the sheath collapse at $180 \mathrm{~ns}$, although $j_{e} \cdot E$ is significantly higher at $180 \mathrm{~ns}$.

In Fig. 2(a) the total absorbed power by the electrons is shown for a variation in the lf voltage amplitude at a fixed power density of $P_{\text {set }}=0.5 \mathrm{~W} / \mathrm{cm}^{2}$. It can be seen, that it is slightly decreasing with a higher admixture of the lf component, indicating that more power is deposited into ions. Nevertheless, the total electron production rate increases by a factor of 3 under variation of $120 \mathrm{~V}$ of the lf voltage amplitude. With higher if admixtures the sheath width is increased at a constant power density leading to faster sheath motion, which increases the local and momentary power absorbed by the electrons during sheath expansion and collapse. This results in a significant change in the total electron production rate due to the nonlinear electron impact excitation. However, the time averaged electron density in the plasma bulk $\left(n_{e}\right)$ [Fig. 2(b)] increases by a factor of 1.2 only. This comparatively small gain of electrons indicates that the loss of charged particles is also significantly increased. The main loss of electrons is the electron flux to the electrodes $\left(\Gamma_{e}\right)$, which shows the assumed increment [Fig. 2(b)]. In contrary to frequently investigated low pressure plasmas the plasma boundary sheath is highly collisional under atmospheric pressure conditions. Consequently, the ion flux is not conserved within the sheath and higher sheath voltages result in higher ion fluxes and associated increased losses. This almost compensates the raised electron production within the plasma volume. 




FIG. 3. Time and space resolved electron impact excitation of helium for a lf component of $V_{\mathrm{lf}}=80 \mathrm{~V}$. (a) $\alpha-\gamma$ hybrid mode operation at $P_{\text {set }}$ $=6 \mathrm{~W} / \mathrm{cm}^{2}$ and (b) $\gamma$-mode operation at $P_{\text {set }}=12 \mathrm{~W} / \mathrm{cm}^{2}$.

Increasing the externally set power density coupled into the plasma leads to mode transitions as already reported for single frequency plasmas in Ref. 16. In the $\alpha$ - $\gamma$ hybrid mode at $P_{\text {set }}=6 \mathrm{~W} / \mathrm{cm}^{2}$ a third excitation structure, additionally to the sheath expansion and collapse structures, within the sheath region is observed [Fig. 3(a)]. These structures arising from electrons accelerated in the sheath electric field, causing an electron avalanche. The initial electrons in the sheath region are produced by Penning ionization and secondary electron emission from the electrodes. During each $14 \mathrm{MHz}$ $(\mathrm{T}=71.4 \mathrm{~ns})$ cycle one avalanche structure can be observed at each electrode. The highest rate is at maximum sheath expansion of the $2 \mathrm{MHz}(\mathrm{T}=500 \mathrm{~ns})$ cycle. These additional structures start to dominate under further increment of the power density, which can be seen in Fig. 3(b) at $P_{\text {set }}$ $=12 \mathrm{~W} / \mathrm{cm}^{2}$. Here the plasma operates in $\gamma$-mode, driven by the high transient electric fields present in the plasma sheath, which are determined by the total applied voltage. In particular in this operation mode it is essential to set a sinusoidal voltage to be able to capture the very strong generation of higher harmonics in the current density.

The mode transitions between the three distinct modes: $\alpha$-, hybrid, and $\gamma$-mode are shown in Fig. 4 . Here the hf voltage amplitude is plotted versus the bulk electron density for different lf voltage admixtures $\left(V_{\mathrm{lf}}=0,40,80\right.$, and 120 V). It can be observed that the plotted curves all have a region with positive slope at low electron densities, as well as a region with negative slope at higher electron densities. The switch to a negative slope indicates the mode transition from the hybrid mode to the $\gamma$-mode. The transition point from $\alpha$-mode to the hybrid mode can be found by comparison of the maximum electron impact excitation rates within the plasma sheath and within the plasma bulk. We define the transition at the power density where both rates are equal. The transitions between the three different operating regimes are indicated in Fig. 4 by dashed lines. These mode transitions are highly influenced by the frequency coupling of the two applied sinusoidal voltages.

We showed that $2 \mathrm{f}$ operation provides enhanced control of the $\alpha-\gamma$ mode transitions as well as the electron dynamics.

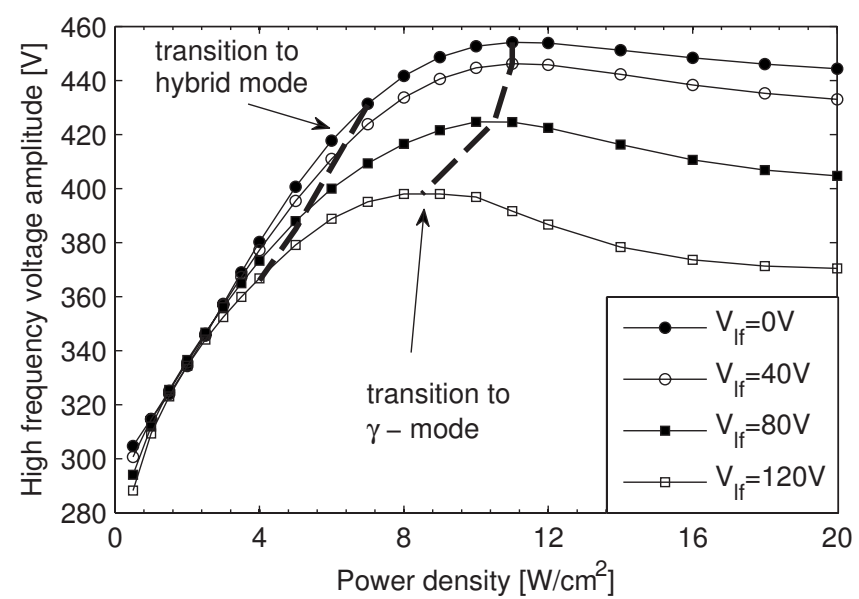

FIG. 4. Hf voltage amplitude of the $2 \mathrm{f}$ excitation signal vs power density. Plotted for several constant if voltage amplitudes.

The dynamics of plasma ionizations is more critically governed by the power absorbed per electron rather than the total power. It is also revealed that electron production on the one hand is significantly enhanced with dual frequency operation, however losses on the other hand are also increased in the collision dominated plasma boundary sheaths.

This work has been supported by Y. Sakiyama and D. B. Graves from University California Berkley. This work is funded through a Science and Innovation Award of EPSRC (Grant No. EP/D06337X/1).

${ }^{1}$ M. M. Turner and P. Chabert, Phys. Rev. Lett. 96, 205001 (2006).

${ }^{2}$ P. Chabert, P. Levif, J.-L. Raimbault, J.-M. Rax, M. M. Turner, and M. A. Lieberman, Plasma Phys. Controlled Fusion 48, B231 (2006).

${ }^{3}$ E. Kawamura, M. A. Lieberman, and A. J. Lichtenberg, Phys. Plasmas 13 053506 (2006).

${ }^{4}$ H. C. Kim and J. K. Lee, Phys. Rev. Lett. 93, 085003 (2004).

${ }^{5}$ T. Kitajima, Y. Takeo, Z. L. Petrovic, and T. Makabe, Appl. Phys. Lett. 77, 489 (2000).

${ }^{6}$ D. O'Connell, T. Gans, E. Semmler, and P. Awakowicz, Appl. Phys. Lett. 93, 081502 (2008)

${ }^{7}$ T. Gans, J. Schulze, D. O'Connell, U. Czarnetzki, R. Faulkner, A. R. Ellingboe, and M. M. Turner, Appl. Phys. Lett. 89, 261502 (2006).

${ }^{8}$ Z. Donkó and Z. L. Petrovic, Jpn. J. Appl. Phys., Part 1 45, 8151 (2006).

${ }^{9}$ J. J. Shi and M. G. Kong, Phys. Rev. Lett. 96, 105009 (2006).

${ }^{10}$ F. Iza, J. K. Lee, and M. G. Kong, Phys. Rev. Lett. 99, 075004 (2007).

${ }^{11}$ V. Schulz-von der Gathen, L. Schaper, N. Knake, S. Reuter, K. Niemi, T. Gans, and J. Winter, J. Phys. D: Appl. Phys. 41, 194004 (2008).

${ }^{12}$ K. H. Becker, K. H. Schoenbach, and J. G. Eden, J. Phys. D: Appl. Phys. 39, R55 (2006).

${ }^{13}$ A. Fridman, A. Chirokov, and A. Gutsol, J. Phys. D: Appl. Phys. 38, R1 (2005).

${ }^{14}$ Q. Li, J.-T. Li, W.-C. Zhu, X.-M. Zhu, and Y.-K. Pu, Appl. Phys. Lett. 95, 141502 (2009).

${ }^{15}$ Y. Sakiyama and D. B. Graves, J. Phys. D: Appl. Phys. 39, 3644 (2006)

${ }^{16}$ J. J. Shi and M. G. Kong, J. Appl. Phys. 97, 023306 (2005).

${ }^{17}$ G. J. M. Hagelaar and L. C. Pitchford, Plasma Sources Sci. Technol. 14 722 (2005).

${ }^{18}$ D. W. Liu, F. Iza, and M. G. Kong, Appl. Phys. Lett. 93, 261503 (2008)

${ }^{19}$ Yu. B. Golubovskii, V. A. Maiorov, J. Behnke, and J. F. Behnke, J. Phys. D: Appl. Phys. 36, 39 (2003).

${ }^{20}$ Y. Sakiyama and D. B. Graves, J. Appl. Phys. 101, 073306 (2007).

${ }^{21}$ A. Yanguas-Gil, K. Focke, J. Benedikt, and A. von Keudell, J. Appl. Phys. 101, 103307 (2007).

${ }^{22}$ X. Yuan and L. L. Raja, IEEE Trans. Plasma Sci. 31, 495 (2003).

${ }^{23}$ A. Salabaş and R. P. Brinkmann, Jpn. J. Appl. Phys., Part 1 45, 5203 (2006). 